\title{
Aconitine linoleate, a natural lipo-diterpenoid alkaloid, stimulates anti-proliferative activity reversing doxorubicin-resistant in MCF-7/ADR breast cancer cells as a selective topoisomerase Ila inhibitor
}

\section{Shangxian Luan}

Sichuan Agricultural University

\section{Yingying Gao}

Sichuan Agricultural University

Xiaoxia Liang ( $\square$ liangxiaoxia@sicau.edu.cn )

Sichuan Agricultural University https://orcid.org/0000-0001-5002-5327

\section{Li Zhang}

Sichuan Agricultural University

\section{Qiang Wu}

Sichuan Agricultural University

\section{Yunkai Hu}

Sichuan Agricultural University

Lizi Yin

Sichuan Agricultural University

Changliang He

Sichuan Agricultural University

Shixi Liu

Yunnan University

\section{Research Article}

Keywords: Aconitine linoleate, cytotoxic activity, topoisomerase lla inhibitor, acute toxicity, MCF-7 and MCF-7/ADR breast cancer cells, Molecular modeling

Posted Date: October 22nd, 2021

DOI: https://doi.org/10.21203/rs.3.rs-1005235/v1 
License: (c) (i) This work is licensed under a Creative Commons Attribution 4.0 International License. Read Full License 


\section{Abstract}

Aconitine linoleate (1) is a lipo-diterpenoid alkaloid, isolated from Aconitum sinchiangense W. T. Wang. The study aimed at investigating the anti-proliferative efficacy and the underlying mechanisms of 1 against MCF-7 and MCF-7/ADR cells, as well as obvious the safety evaluation in vivo. The cytotoxic activities of 1 were measured in vitro. Also, we investigated the latent mechanism of 1 by cell cycle analysis in MCF-7/ADR cells, and Topo I, Topo lla inhibition assay. Molecular docking is done by Discovery Studio 3.5 and Autodock vina 1.1.2. Finally, the acute toxicity of 1 was detected on mice. 1 exhibited significant anti-tumor activity against both MCF-7 and MCF-7/ADR cells, with IC ${ }_{50}$ value of 7.58 and $7.02 \mu \mathrm{M}$, which is 2.38 times and 5.05 times more active, respectively than etoposide in both cell lines, and being 9.63 times more active than adriamycin in MCF-7/ADR cell lines. The molecular docking and topo inhibition test found that it's a selective inhibitor of topoisomerase $\llbracket a$. Moreover, activation of damage response pathway of the DNA leads to cell cycle arrest at $\mathrm{G}_{0} \mathrm{G}_{1}$ phase. Furthermore, the in vivo acute toxicity of 1 in mice displayed lower toxicity than aconitine, with $\mathrm{LD}_{50}$ of $2.2 \times 10^{5} \mathrm{nmol} / \mathrm{Kg}$ and only slight pathological changes in liver and lung tissue, 489 times safer than aconitine. In conclusion, compared with aconitine, 1 has more significant anti-proliferative activity against MCF-7 and MCF-7/ADR cells, and greatly reduces in vivo toxicity, which suggesting this kind of lipo-alkaloids are powerful and promising antitumor compounds for breast cancer.

\section{Introduction}

According to the statistical data from the World Health Organization (WHO) in 2020, breast cancer has taken the top one place both in diagnose rate and mortality rate for female (Sung et al., 2021). However, its treatment has been hindered by multidrug resistance (MDR), leading to the urge for new antitumor agent targeted specific target, especially for reversing the resistant problems. Among the various anticancer targets, DNA topoisomerases (topos) are well-known ones (Li and Liu, 2001, Pommier, 2006, McClendon and Osheroff, 2007, Nitiss, 2009b, Nitiss, 2009a). Topos inhibitors are generally classified into Topo I inhibitors as camptothecin (CPT) (Schoeffler and Berger, 2008), and Topo II inhibitors as amsacrine, etoposide and doxorubicin (Khadka and Cho, 2013). However, the clinic use of current anticancer topoisomerase inhibitors have been limited by some major negative consequences. Firstly, most clinically active topo inhibitors are topo poisons, such as etoposide and doxorubicin, which usually need monitoring for the severe genetic toxicity (Jun et al., 2015). Secondly, the severe dose-limiting toxicities and drug resistance of Topo I inhibitors like CPT have been emerged. Thirdly, the secondary malignancies led by the inhibition of Topo II $\beta$ in the clinic use of the unselective Topo II inhibitors have been reported (Felix, 1998, Mistry et al., 2005, Azarova et al., 2007). For these reasons, many researchers have targeted Topo Ila, rather than $I / \beta$, on developing topo catalytic inhibitors, which induces cancer cell death through the elimination of the essential enzymatic activity, rather than topo poisons(Albert et al., 2013, Liang et al., 2019). 
As a widely used traditional Chinese medicine, Aconitum possesses anti-inflammatory, analgesic, and anti-cancer activities (Kim et al., 2002, Liang et al., 2016b). Single Aconitum and its prescriptions have been documented for the treatment of cancer since last century in China (Mingxin Tang and Sun, 1986, He Shu et al., 2009, Chen, 2015, Qiuping Xu et al., 2016, Duan et al., 2018). However, the precise mechanisms of the Aconitum eliciting anticancer effects have not yet been fully clarified due to the diverse structures of compounds in Aconitum. Diterpenoid alkaloids, the main active components from Aconitum, have been reported as potential cytotoxic agents for decades (Li et al., 2005, Hazawa et al., 2009a, Hazawa et al., 2009b, Wada et al., 2011, Wada, 2012, Wada et al., 2012, Wada et al., 2015a, Wada et al., 2015b, Liang et al., 2016a, Liang et al., 2017, Liang et al., 2018, Wada K., 2019) (Figure 1). But the significant toxicities of diterpenoid alkaloids, such as the neurotoxicity and cardiotoxicity of aconitine (4), limited their further clinical application. Lipo-diterpenoid alkaloids, a kind of diterpenoid alkaloids bearing long ester groups (such as 1-3 in Figure 1), are common components obtained from Aconitum, which have been reported with extensive pharmacological activities and low toxicity (Kitagawa et al., 1982, Isao et al., 1984, Hanuman and Katz, 1994, Yamashita et al., 2018, Yamashita et al., 2020). In 2017, our group first reported the obvious cytotoxic activities of lipo-diterpenoids 1 and 3, against HL-60, A-549, SMCC7721, MCF-7 and SW480 cell lines, comparable to cisplatin (Liang et al., 2016a, Liang et al., 2017). Later, the good cytotoxic activities of one lipo-alkaloid against several tumor cell lines (A549, MDA-MB-231, MCF-7, KB, KB-VIN) also have been reported by Koji Wada group (Koji Wada, 2019, Yamashita H., 2020). Then, the highly selective index $(\mathrm{SI}>10)$ for compound 1 between MCF-7 and mouse fibroblast cells, has surprised us, arousing a deeper pharmacological mechanism research in current study.

In this work, aconitine linoleate (1) was investigated for its anti-proliferative activity in vitro against MCF-7 and adramycin resistant subline MCF-7/ADR cell lines. In order to explore its mechanistic pathways, the lead compound 1 was performed extra investigations such as cell cycle analysis, topoisomerase I / II inhibition, and molecular docking. Meanwhile, for determining its safety the acute toxicity on mice was also tested.

\section{Materials And Methods}

\subsection{Materials and reagents}

Aconitine linoleate (1) (isolated from the Aconitum sinchiangense W. T. Wang, plants were collected from Yili Kazakh Autonomous Prefecture, Xinjiang Uygur Autonomous Region, China, in 2016, and was identified by Dr. Lixia Li. The plants are Stored in the Natural Medicine Research Center of Sichuan Agricultural University, the voucher specimen No. 20140616-1); MCF-7 cell line (Breast cancer cell MCF-7), MCF-7/ADR (adramycin resistant subline) and NIH3T3 cell line (Mouse fibroblast cells) (Cell Resource Center, Shanghai Institutes for Biological Sciences, Chinese Academy of Sciences); Kuming mice (CHENGDU DOSSY EXPERIMENTAL ANIMALS), PBS (HyClone); DMEM high glucose (HyClone); fetal bovineserum (FBS) (Gibco); propidium iodide (PI) (Yeasen Biotech); Adriamycin (RYON); Etoposide and hydroxy camptothecin (TargetMol);Trypsin-EDTA (0.25\%), phenol red (solarbio); Penicillin-Streptomycin Liquid (solarbio); CellTiter 96®AQueous One Solution Cell Proliferation Assay (Promega); Paraffin wax 
(Shanghai Hualing kangfu Machinery Factory); Ehrlich hematoxylin stain and Eosin Solution (solarbio); LDH Cytotoxicity Assay Kit (solarbio); Prestained Protein Ladder, 10 to 180 kDa and 10-170 kDa, RIPA Lysis Buffer, Topoisomerase $₫$ alpha Rabbit Monoclonal Antibody, Topoisomerase $\otimes$ Rabbit Monoclonal Antibody, BeyoECL Moon, SDS-PAGE Sample Loading Buffer (5X), TOP2B Rabbit Polyclonal antibody (PROTEINTECH GROUP); Goat Anti-Rabbit IgG, HRP Conjugated (Wuhan Saiweier Biological Technology); pBR322 DNA, DNA Topoisomerase I (Takara Bio); PVDF Membrane(Biotopped); DNA Topoisomerase Ila (TopoGEN).

All animal experiments were approved by the guidelines of Animal Ethical and Welfare Committee (\#20190036) in Sichuan Agricultural University. And the animals were treated according to the Guide for the Care and Use of Laboratory Animals (Eighth Edition, 2011, The National Academies Press).

\subsection{In vitro anti-proliferative activity}

Taking adriamycin and etoposide as the positive controls, the cytotoxic activities of aconitine linoleate (1) were measured in vitro on MCF-7 cell line (Breast cancer cell MCF-7) and MCF-7/ADR (adramycin resistant subline) using MTS Cell Proliferation Colorimetric Assay Kit (Abdelhaleem et al., 2018, Luan et al., 2021). The cytotoxicity test sample (purity greater than 95\%) was dissolved in $1 \% \mathrm{HCl}$ and adjusted the $\mathrm{pH}$ value to 6-7 with $1 \mathrm{~mol} / \mathrm{L} \mathrm{NaOH}$, then configured into a series of drug concentrations $(150,75$, $37.5,18.75$, and $9.375 \mu \mathrm{g} / \mathrm{mL}$ ). Take logarithmic growth phase MCF-7 cells, MCF-7/ADR cells, digest and count them with trypsin, prepare a cell suspension with a concentration of $5 \times 10^{4}$ cells $/ \mathrm{mL}$ with the culture medium, and add $100 \mu \mathrm{L}$ of cell suspension to each well of a 96 -well cell culture plate (Approximately $5 \times 10^{3}$ cells per well). Place the 96 -well cell culture plate in a $37{ }^{\circ} \mathrm{C}, 5 \% \mathrm{CO}_{2}$ incubator for $24 \mathrm{~h}$. Then culturing these cells for 48 hours with the prepared drugs at various concentrations, MTS solution ( $20 \mu \mathrm{L}, 2.5 \mathrm{mg} / \mathrm{mL}$ in PBS) and $180 \mu \mathrm{L}$ of culture medium were added for continuous incubation for $2.5 \mathrm{~h}$, then measured at $490 \mathrm{~nm}$ for the light absorption value. The cell growth inhibition rate was calculated with the equation: Survival fraction $=$ O.D. (treated cells)/O.D. (control cells). The relation between surviving fraction and compound concentration was plotted and $\mathrm{IC}_{50}$ (the concentration required for $50 \%$ inhibition of cell viability) was calculated for each test compound. In order to further explore the selectivity between 1 and normal cells, we tested NIH3T3 cell line (Mouse fibroblast cells) in the same way. (Except that the drug concentration was set to 380, 190, 95, 47.5, $23.75 \mu \mathrm{g} / \mathrm{mL}$ )

\subsection{In vitro cell cycle analysis}

After treating with compound 1 for 48 hours, the MCF-7 cells were washed twice with ice-cold phosphate buffer saline (PBS). The cells was obtained after centrifugation for 5 minutes $(1000 \mathrm{r} / \mathrm{min})$, and fixed with $70 \%$ ethanol for 6 hours at $4^{\circ} \mathrm{C}$, then washed with PBS, re-suspended with $0.1 \mathrm{mg} / \mathrm{mL}$ RNase, stained with $40 \mathrm{mg} / \mathrm{mL}$ propidium iodide (PI), and analyzed by flow cytometry using FACS Calibur (Becton Dickinson) (Schmitta et al., 2019). The cell cycle distributions were calculated using Flowjo.7.6. and SPSS 12.0 software (Becton Dickinson). The interference with the normal cell cycle distribution was indicated after exposure of MCF-7 cells to this compound.

\subsection{Topo inhibition assay}




\subsubsection{Topo I inhibition assay}

Taking hydroxy camptothecin as positive control, reaction mixtures $(20 \mu \mathrm{L})$ with supercoile pBR322 DNA $(0.25 \mu \mathrm{g}$ in the $2 \mu \mathrm{L} 10 \times$ Tris/Glycine/SDS (TGS) buffer), 1 unit Topo I, $2 \mu \mathrm{L} 0.1 \% \mathrm{BSA}$, and $0.2 \mu \mathrm{L}$ aconitine linoleate (1) with different concentrations and appropriate distilled deionized water were prepared. After incubating at $37^{\circ} \mathrm{C}$ for 30 minutes, $10 \%$ SDS $(1 \mu \mathrm{L})$ was added to stop the reaction. A 20 $\mu \mathrm{L}$ aliquot was electrophoresed in $1.2 \%$ agarose gel at $80 \mathrm{~V}$ for $20 \mathrm{~min}$ in $0.5 \times$ Tris-acetate (TBE) buffer (20 mM Tris-acetate, $0.5 \mathrm{mM}$ EDTA, pH 8.3), then at $130 \mathrm{~V}$ for $25 \mathrm{~min}$. After electrophoresis, DNA bands were stained in ethidium bromide $(0.5 \mu \mathrm{g} / \mathrm{mL})$ and visualized by the Bio-Rad gel imaging system.

\subsubsection{Topo Ila inhibition assay}

Taking etoposide as positive control, reaction mixtures $(20 \mu \mathrm{L})$, containing $0.15 \mu \mathrm{g}$ of supercoile pBR322 DNA in the $4 \mu \mathrm{L} 10 \times$ Tris/Glycine/SDS (TGS) buffer (50 mM spermidine, $720 \mathrm{mM} \mathrm{KCl}, 350 \mathrm{mM}$ Tris-HCl pH8.0, $50 \mathrm{mM} \mathrm{MgCl}_{2}, 50 \mathrm{mM}$ DTT), 5 units Topo Ila, $0.2 \mu \mathrm{L}$ aconitine linoleate (1) with different concentrations and appropriate distilled water, were obtained. After incubating at $37^{\circ} \mathrm{C}$ for 30 minutes, $10 \%$ SDS $(1 \mu \mathrm{L})$ was added to stop the reaction. An aliquot $(20 \mu \mathrm{L})$ was electrophoresed in $1.2 \%$ agarose gel at $80 \mathrm{~V}$ for $20 \mathrm{~min}$ in $0.5 \times$ Tris-acetate (TBE) buffer ( $20 \mathrm{mM}$ Tris-acetate, $0.5 \mathrm{mM}$ EDTA, pH 8.3), then at $130 \mathrm{~V}$ for $25 \mathrm{~min}$. After electrophoresis, DNA bands were stained in ethidium bromide $(0.5 \mu \mathrm{g} / \mathrm{mL})$ and visualized by the Bio-Rad gel imaging system.

\subsection{Molecular modeling}

\subsubsection{Molecular Docking}

DS BIOVIA Discovery Studio 3.5 and Autodock vina 1.1.2 were used to conduct the molecular docking studies for the binding mode between compound 1 and Topo lla (Trott and Olson, 2010). The threedimensional (3D) structure of Topo lla (PDB ID: 5GWK) was obtained from the protein database (http://www.rcsb.org/pdb/home/home.do). The 3D structure of the compound was drawn by ChemBioDraw Ultra 14.0 and ChemBio3D Ultra 14.0 software. The docking input files were generated by the AutoDockTools 1.5.6 package (Sanner, 1999, Morris et al., 2009). Ligand structures were prepared for docking by merging non-polar hydrogen atoms and defining rotatable bonds. The search grid of the Topo Ila was identified as center_x: 23.7, center_y: -38.695, and center_z: -60.34 with dimensions size_x: 15 , size_y: 15, and size_z: 15 . The exhaustive value was set to 20 for improving docking accuracy. For Vina docking, the default parameters were used if it was not mentioned. Then an MD study was performed to revise the docking result.

\subsubsection{Molecular Dynamics simulation}

The AmberTools 15 programs and Amber 14 (Götz et al., 2012, Pierce et al., 2012, Salomon-Ferrer et al., 2013) were used for MD simulations of the selected docked pose. The compound was first prepared by the tool, ACPYPE (da Silva and Vranken, 2012), basing on ANTECHAMBER (Wang et al., 2004, Wang et al., 2006) for generating automatic topologies and parameters in different formats for different molecular 
mechanics programs, such as calculation of partial charges. Then, the forcefield "leaprc.gaff" (generalized amber forcefield) was used for preparing the ligand, while "leaprc.ff14SB" was used for the receptor. The system was placed in a rectangular box (with a 10.0 ^ boundry) of TIP3P water using the "SolvateOct" command with the minimum distance between any solute atoms. PMEMD (Particle Mesh Ewald Molecular Dynamics) module was accelerated by GPU (NVIDIA® Tesla K20c) for a short-term minimization (500 steps per steepest descent and conjugate gradient method), 500 ps heating and 50 ps of density equilibration with weak restraints to reach the equilibrium of solvated complexes. At last, $20 \mathrm{~ns}$ of MD simulations were carried out. All the molecular dynamics were performed on Dell Precision T5500 workstation. The Molecular Mechanics/Generalized Born Surface Area (MM/GBSA) method, implemented in AmberTools 15, was used for calculating the the binding free energies $\left(\Delta G_{b i n d}\right.$ in $\mathrm{kcal} / \mathrm{mol}$ ). Moreover, to identify the key protein residues responsible for the ligands binding process, the binding free energy was decomposed on a per-residue basis. The binding free energy of MM/GBSA for each complex, was estimated by following equations: $\Delta G_{\text {bind }}=G_{\text {complex }}-G_{\text {protein }}-G_{\text {ligand }}$ (1) $\Delta G_{\text {bind }}=\Delta H-$ $\mathrm{T} \Delta \mathrm{S} \approx \Delta \mathrm{G}_{\text {gas }}+\Delta \mathrm{G}_{\text {sol }}-\mathrm{T} \Delta \mathrm{S}$ (2) $\Delta \mathrm{G}_{\mathrm{gas}}=\Delta \mathrm{E}_{\text {ele }}+\Delta \mathrm{E}_{\mathrm{vdw}}$ (3) $\Delta \mathrm{G}_{\mathrm{sol}}=\Delta \mathrm{E}_{\mathrm{GB}}+\Delta \mathrm{G}_{\mathrm{SA}}$ (4) (where $\Delta \mathrm{G}_{\text {bind }}$ is the binding free energy and $G_{\text {complex, }} G_{\text {protein }}$ and $G_{\text {ligand }}$ are the free energies of complex, protein, and ligand, respectively. The sum of entropy contribution $(T \Delta S)$ and enthalpy $(\Delta H)$ are for the binding free energy. The enthalpy $(\Delta \mathrm{H})$ includes the free energy of solvation $\left(\Delta \mathrm{G}_{\text {sol }}\right)$ and free energy of the gas phase $\left(\Delta \mathrm{G}_{\text {gas }}\right)$. $\Delta \mathrm{G}_{\text {sol }}$ is composed of $\Delta \mathrm{G}_{\mathrm{SA}}$, the nonelectrostatic solvation component (nonpolar contribution) and $\Delta \mathrm{G}_{\mathrm{GB}}$, the electrostatic solvation energy (polar contribution). $\Delta \mathrm{G}_{\text {gas }}$ includes $\Delta \mathrm{E}_{\text {ele }}$ (electrostatic) and $\Delta \mathrm{E}_{\mathrm{vdw}}$ (van der Waals) energies, which exhibits the gas-phase interaction energy between protein and ligand.)

\subsection{Acute toxicity}

The acute toxicity of the lipo-diterpenoid alkaloids to animal subjects were investigated on the normal mice by using the aconitine linoleate (1). Briefly, 110 kuming mice (aged 4-5 weeks, weighed 18-22 g, sex in half) were divided randomly into 11 groups, including one negative control group (dilute hydrochloric acid solution, pH 5.4), five dose groups $(220,210,200,190,180 \mathrm{mg} / \mathrm{Kg})$ of aconitine linoleate, and five dose groups $(0.6,0.55,0.5,0.4,0.3 \mathrm{mg} / \mathrm{Kg})$ for the positive control aconitine. All the groups were given the samples by intraperitoneal injection once time. The animals were observed for toxic signs for $4 \mathrm{~h}$ after the drug injection. And the changes in physical appearance, injury, pain and signs of illness were recorded daily for the 14 days. Finally, the mice were sacrificed after 14 days and the major tissues, including hart, spleen, kidney, liver, lung and thymus were excised for the analysis of macroscopic pathologicalanatomy. Then the tissues, fixed in formaldehyde $37 \%$, were taken for microscopic evaluation using haematoxylin and eosin staining in the Pathology Laboratory of College of veterinary medicine, Sichuan Agricultural University. The pathological changes of the tissues were assessed and compared.

\section{Results}

\subsection{Biology}


Taking adriamycin and etoposide as positive control, the in vitro anti-proliferative activity of Aconitine linoleate (1) was examined against MCF-7 and MCF-7/ADR cell lines by MTS method (Ahmed et al., 2017). The results were expressed as $I C_{50}$ (median growth inhibitory concentration) values, representing the concentrations needed to produce a $50 \%$ inhibition of cell growth after incubation for $48 \mathrm{~h}$ (Table 1 and Figure 2) (Luan et al., 2021). In order to further explore the selectivity between 1 and normal cells, we used $\mathrm{NIH} 3 \mathrm{~T} 3$ cell line as normal cells for comparison and found that the $\mathrm{IC}_{50}$ was $72.08 \pm 0.15 \mu \mathrm{M}$, and selective index $(\mathrm{SI})=9.51$ and 10.26 respectively compared with MCF-7 and MCF-7/ADR (Table 1).

To explore the effect of the Aconitine linoleate on cell proliferation of MCF-7/ADR cells, we analyzed cell cycle distribution of aconitine linoleate (1) at its $\mathrm{IC}_{50}$ concentration (Table 1 ) using flow cytometry assay. Our data in Figure 3 shows that aconitine linoleate (1), containing 8-linoleic acid side chain, $3-\mathrm{OH}$ and $\mathrm{N}$ ethyl group, exhibits the potent anti-proliferative effect on MCF-7/ADR cells by increasing percentage of cells $G_{0} G_{1}$ phase, decreasing the same in $S$ phase.

\subsection{Molecular docking}

To better understand the potential binding targets of lipo-diterpenoid alkaloids, the molecular docking of aconitine linoleate (1) with 18 kinds of common antitumor targets was first screened by Discovery Studio 3.5.

The docking results in Table 2, indicated the most favorable target would be Topo II for the highest Libdockscore (324.932). Interestingly, Topo lla may be the selective target (Libdockscore of 102.535), rather than Topo $\| \beta$, which has been failed in docking process. In contrast, aconitine (4) could not successfully dock with those two targets (Table 3), relating to the bad anti-proliferative activity of aconitine against those two cancer cells.

The potential binding mode between compound $\mathbf{1}$ and Topo lla has been further explored by molecular docking and molecular dynamic simulations, using the AutoDock vina 1.1.2 and Amber14 software. Bonding models of 1 and Topo lla were depicted in Figure 4, showing the residue A/Arg-487 close to the phenyl group of the compound 1 by cation-r interaction (Figure 4A). To gain more information about the residues surrounding the binding site and their contribution to the whole system, the MMGBSA approach was used to calculate the contribution of the electrostatic, solvation, Van der Waals force, and residues to the free energy of binding. Each residue interaction free energy was presented as electrostatic $\left(\Delta E_{e l e}\right)$, solvation $\left(\Delta E_{s o l}\right)$, Van der Waals $\left(\Delta E_{v d w}\right)$ and total contribution $\left(\Delta E_{\text {total }}\right)$. In the Topo lla-1 complex, the residue $\mathrm{A} / \mathrm{Arg}-487$ has a strong electrostatic $\left(\Delta E_{\text {ele }}\right)$ contribution, with the $\Delta E_{\text {ele }}$ of $<-11.0$ $\mathrm{kcal} / \mathrm{mol}$ (Figure 4B). In addition, due to the close proximity between the nucleotides and the compound 1, strong Van der Waals interactions (<-4.5 kcal/mol) between nucleotides C/DC-8, D/DT-9, F/DA-12 and F/DG-13, and the ligand have been detected. Overall, the majority of the decomposed energy interaction originated from Van der Waals interactions, mainly through hydrophobic interactions, such as A/Met-762, A/Met-766 and B/Ala-801. It referred that the introduction of hydrophobic groups, such as halogen, nitro, benzene ring, etc., into the molecule, may increase the binding force with Topo Ila, thereby enhancing its 
anti-proliferative activity. Furthermore, the calculation of the total binding free energy for the Topo lla-1 complex led to an estimated $\Delta \mathrm{G}_{\text {bind }}$ of $-9.1 \mathrm{kcal} / \mathrm{mol}$ for $\mathbf{1}$ (Table 4), indicating the strongly bind of $\mathbf{1}$ to the binding site of the Topo Ila. This suggests that the anti-proliferative activity of 1 is very likely to take effect through the complex with Topo Ila.

The docking results of 20-ns molecular dynamics simulation confirmed the preferential binding mechanism of Topo lla and 1 . The root mean square deviation (RMSD) values of the protein skeleton basing on the initial structure with the simulation time was performed to figure out the dynamic stability of the models and ensure the rationality of the sampling strategy (Figure $4 \mathrm{C}$ ), which proved the stability of protein in two systems under the simulation. The flexibility of the whole protein residues in Topo lla-1 complex and free Topo lla were detected by the root mean square fluctuations (RMSF) values (Figure 4D), presenting the difference in flexibility between the binding site of Topo lla with 1 . The results presented a small degree of flexibility with a RMSF of less than $3 \AA$ at the binding site, referring these residues seem to be more rigid as a result of binding to 1 . In summary, the rational explanation of the interactions between 1 and Topo lla by the molecular dynamic simulations provided valuable information for further development of the Topo lla inhibitors.

In order to prove the molecular docking results, the activity inhibition of topoisomerase enzyme with compound 1 was suspected as expecting mechanism for the action of lipo-diterpenoid alkaloids. The assay was performed to evaluate the Topo I, Ila inhibitory activity (Figure 5). Aconitine linoleate (1) exhibited promising inhibition activity below $200 \mu \mathrm{M}$, comparable to the known potent Topo Ila inhibitor, etoposide. Whereas, it exhibited no obvious inhibition against Topo I, even at the concentration of 200 $\mu \mathrm{M}$. Thus, it was proved that compound 1 exerted its anti-proliferative activity through selective Topo lla inhibition, basing the results of both molecular docking and inhibition of topoisomerase activity. However, in the Topo Ila inhibition assay, the $\mathrm{IC}_{50}$ values were much higher than in the cytotoxicity assays. It can be concluded that compound 1 possessed the anti-proliferative activity partially dependent on the Topo Ila inhibition.

\subsection{Acute toxicity}

For further application, the toxicity evaluation should be explored as an important factor. To investigate the toxicity of lipo-diterpenoid alkaloids, the acute toxicity test of aconitine linoleate (1) was carried out in Kuming mice for the $L D_{50}$ (half lethal dose) value. The results showed that the $L_{50}$ value of aconitine linoleate (1) was $2.2 \times 10^{5} \mathrm{nmol} / \mathrm{Kg}$. Compared with aconitine $\left(\mathrm{LD}_{50}=4.5 \times 10^{2} \mathrm{nmol} / \mathrm{Kg}\right)$, the safe dosage of aconitine linoleate to mice is 489 times higher than aconitine, suggesting the obvious toxicity reduction for lipo-diterpenoids (Table 5). Furthermore, as shown in Figure 6, after aconitine linoleate treatment, no obvious pathological lesions appear in the main tissues, including hart, spleen, kidney, liver, lung and thymus. The pathological morphology in mice tissue with/without compound $1\left(2.2 \times 10^{5}\right.$ $\mathrm{nmol} / \mathrm{Kg})$ or aconitine $\left(4.5 \times 10^{2} \mathrm{nmol} / \mathrm{Kg}\right)$ were also conducted by H\&E staining method. Compared with the blank group, only liver and lung tissue in the aconitine linoleate group showed slight pathological changes, which are much milder than the aconitine group. For liver tissue, the obvious damage is found 
in the aconitine group with moderate diffuse vacuolar degeneration and punctate necrosis foci, which are occasionally seen in the aconitine linoleate group. For lung tissue, the alveolar septum is significantly thicker and the number of alveoli is decreased in the aconitine group; whereas, the alveolar septum in the aconitine linoleate group is slightly thicker, significantly better than that of the aconitine group.

\section{Discussion}

Firstly, compared with aconitine (4), aconitine linoleate (1) exhibited high in vitro cytotoxic activities against both MCF-7 and MCF-7/ADR cell lines, and displayed much lower toxicity in the in vivo acute toxicity evaluation in mice. Based on the results of molecular docking, aconitine linoleate (1) selectively well docked with Topo Ila, while aconitine (4) failed. Considering the tumor targeting effect of long chain fatty acids (Sauer et al., 2000), it is concluded that the introduction of 8-lipo group significantly enhanced its affinity and selectivity for Topo lla, as well as increasing the tumor targeting which led to the low toxicity.

Secondly, according to the data of flow cytometry assay, aconitine linoleate (1) exhibits cytotoxic effect on MCF-7/ADR cells by increasing percentage of cells G0/G1 phase, decreasing the same in S phase. As everyone knows, Topo Ila and $I / \beta$ are two types of Topo II in humans. The expression level of Topo Ila is the highest in G2/M phase, followed by $S$ phase, and lowest in G0/G1 phase, but that of Topo II $\beta$ is always constant (Jun et al., 2015). Therefore, due the selective inhibition of Topo Ila, aconitine linoleate (1) targeted the tumor cells in $S$ phase.

Thirdly, as a widely used chemotherapeutic agent, doxorubicin, mainly targeting Topo Ila (Du et al., 2011), has caused drug-resistance during clinical application, which is a complex process (Ganapath and Ganapathi, 2013). Different resistant mechanisms have been recognized, including the enhanced expression of the P-glycoprotein, ABCB1, ABCG2, the down-expression level of topo lla, overexpression of multidrug resistance protein 1(MDR1), and even the activation of autophagy (Mechetner et al., 1998, Latorre et al., 2012, Shatha AbuHammad and Zihlif, 2013, Guo et al., 2016). Although targeted to the same enzyme, aconitine linoleate (1) is mainly a topo catalytic inhibitor, which could selectively inhibit Topo Ila, rather than a topo poison like doxorubicin. They may have different topo-inhibit mechanism. Moreover, the reversal mechanism of tumor drug resistance of aconitine linoleate (1) should be a multitargeted way, which need further explore in the next step.

\section{Conclusions}

We conclude that aconitine linoleate (1) not only selectively inhibits the expression and activity of Topo Ila, but also induce a significant increase in the percentage of cells at $G_{0} G_{1}$ phase, leading its excellent anti-proliferative activity against MCF-7 and MCF-7/ADR in vitro. Since the introduction of 8-lipo group, 1 is much less toxic than aconitine. Lipo-alkaloid would be a powerful and potentially anti-tumor compound against drug-resistant breast cancer, bringing more possibilities for fighting breast cancer in the future. 


\section{Associated Content}

Supporting Information

The Supporting Information is available free of charge on website at DOI:

Data availability

As supplemental material and upon request.

Accession Codes

The PDB access code for structure of 1 bond to topoisomerase lla is 5GWK. Authors will release the atomic coordinates and experimental data upon article publication.

\section{Declarations}

Competing interests

The authors declare that they have no known competing financial interests or personal relationships that could have appeared to influence the work reported in this paper.

Ethics approval and consent to participate

The animal use protocol listed below has been reviewed and approved by the Sichuan Agricultural University Animal Ethical and Welfare Committee.

Consent for publication

All authors approved the manuscript to be published.

\section{Author contributions}

Shangxian Luan: Writing paper and participating in Molecular Docking and in vitro anti-proliferative activity, acute toxicity. Yingying Gao: Participating in in vitro cell cycle analysis and Topo inhibition assay. Xiaoxia Liang: Designing and leading experiments, writing and revising the paper. Li Zhang: participating in acute toxicity. Qiang Wu: participating in acute toxicity. Yunkai Hu: participating in acute toxicity. Shixi Liu: participating in Molecular Docking. Lizi Yin and Changliang He guided the experiment. The authors declare that all data were generated in-house and that no paper mill was used.

\section{Funding}

This study was supported by the National Natural Science Foundation of China Youth Fund and the National Natural Science Foundation of China (Grant No. 81703387, 31972743) 


\section{References}

Abdelhaleem EF, Abdelhameid MK, Kassab AE, Kandeel MM (2018) Design and synthesis of thienopyrimidine urea derivatives with potential cytotoxic and pro-apoptotic activity against breast cancer cell line MCF-7. Eur J Med Chem 143: 1807-1825. https://doi.org/10.1016/j.ejmech.2017.10.075

Ahmed MS, Elsenduny FF, Taylor J, Halaweish FT (2017) Biological screening of cucurbitacin inspired estrone analogs targeting mitogen-activated protein kinase (MAPK) pathway. Chem Biol Drug Des 90: 478-484. https://doi.org/110.1111/cbdd.12963

Albert B, Gibalova L, Seres M, Barancik M, Sulova Z (2013) New insight into p-glycoprotein as a drug target. Anti-cancer Agents Med Chem 13: 159-170. https://doi.org/10.2174/1871520611307010159

Azarova AM, Lyu YL, Lin C-P, Tsai Y-C, Lau JY-N, Wang JC, Liu LF (2007) Roles of DNA topoisomerase II isozymes in chemotherapy and secondary malignancies. Proceedings of the National Academy of Sciences 104: 11014-11019. https://doi.org/10.1073/pnas.0704002104

Chen G (2015) Clinical Observation and Experimental Study on the Treatment of Breast Cancer with the Method of Warming Yang and Dissipating Phlegm Beijing University of Chinese Medicine

da Silva AWS, Vranken WF (2012) ACPYPE-Antechamber python parser interface. BMC research notes 5: 367. https://doi.org/10.1186/1756-0500-5-367

Du Y, Zhou Q, Yin W, Zhou L, Di G, Shen Z, Shao Z, Lu J (2011) The role of topoisomerase lla in predicting sensitivity to anthracyclines in breast cancer patients: a meta-analysis of published literatures. Breast cancer research and treatment 129: 839. https://doi.org/10.1007/s10549-011-1694-9

Duan F, Lang L, Zhang X, Hao X, Zhang Y, Zhao S (2018) Effect and Complications for Sini Decoction Combined with Western Medicine Treatment on Advanced Breast Cancer after Operation. Chinese Archives of Traditional Chinese Medicine 36: 1774-1776.

Felix CA (1998) Secondary leukemias induced by topoisomerase-targeted drugs. Biochimica et Biophysica Acta (BBA) - Gene Structure and Expression 1400: 233-255. https://doi.org/10.1016/S01674781(98)00139-0

Ganapath RN, Ganapathi MK (2013) Mechanisms regulating resistance to inhibitors of topoisomerase II. Frontiers in pharmacology 4: 89. https://doi.org/10.3389/fphar.2013.00089

Götz AW, Williamson MJ, Xu D, Poole D, Le Grand S, Walker RC (2012) Routine microsecond molecular dynamics simulations with AMBER on GPUs. 1. Generalized Born. Journal of chemical theory and computation 8: 1542-1555. https://doi.org/10.1021/ct200909j

Guo B, Tam A, Santi SA, Parissenti AM (2016) Role of autophagy and lysosomal drug sequestration in acquired resistance to doxorubicin in MCF-7 cells. BMC cancer 16: 762. https://doi.org/10.1186/s12885- 
Hanuman JB, Katz A (1994) New Lipo Norditerpenoid Alkaloids from Root Tubers of Aconitum ferox. Journal of Natural Products 57: 105-115. https://doi.org/10.1021/np50103a015

Hazawa M, Takahashi K, Wada K, Mori T, Kawahara N, Kashiwakura I (2009a) Structure-activity relationships between the Aconitum C20-diterpenoid alkaloid derivatives and the growth suppressive activities of Non-Hodgkin's lymphoma Raji cells and human hematopoietic stem/progenitor cells. Investigational new drugs 29: 1-8. https://doi.org/10.1007/s10637-009-9327-4

Hazawa M, Wada K, Takahashi K, Mori T, Kawahara N, Kashiwakura I (2009b) Suppressive effects of novel derivatives prepared from Aconitum alkaloids on tumor growth. Invest New Drugs 27: 111119. https://doi.org/10.1007/s10637-008-9141-4

He Shu, Xuecai Liu, Jia E (2009) Study on the anti-tumor effects of aconitides World Health Digest Medical Periodieal 6: 258.

Isao K, Zhaolong C, Minoru Y, Katsuya K, Masayuki Y, Naohiko O, Yuji Y (1984) Chemical Studies on Crude Drug Processing. III. Aconiti Tuber (2). : On the Constituents of \"Pao-fuzil", the Processed Tuber of Aconitum carmichaeli DEBX. and Biological Activities of Lipo-alkaloids. Yakugaku Zasshi 104: 858866. https://doi.org/10.1248/yakushi1947.104.8_858

Jun K-Y, Park S-E, Liang JL, Jahng Y, Kwon Y (2015) Benzo [b] tryptanthrin inhibits MDR1, topoisomerase activity, and reverses adriamycin resistance in breast cancer cells. Chem Med Chem 10: 827835. https://doi.org/10.1002/cmdc.201500068

Khadka DB, Cho W-J (2013) Topoisomerase inhibitors as anticancer agents: a patent update. Expert Opin Ther Pat 23: 1033-1056. https://doi.org/10.1517/13543776.2013.790958

Kim S, Lee SR, Ooda H, Lee JI (2002) Pharmacologic evalution of some anti-cancer plants from

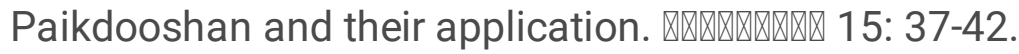

Kitagawa I, Yoshikawa M, Chen ZL, Kobayashi K (1982) Four new lipo-alkaloids from Aconiti tuber. Chem Pharm Bull 30: 758-761.

Koji Wada MG, Takahiro Shimizu, Nami Kusanagi, Megumi Mizukami, Yuji Suzuki, Kang-Po Li, Kuo-Hsiung Lee, Hiroshi Yamashita (2019) Structure-activity relationships and evaluation of esterified diterpenoid alkaloid derivatives as antiproliferative agents. Journal of Natural Medicines 73: 789799. https://doi.org/10.1007/s11418-019-01331-6

Latorre E, Tebaldi T, Gabriella Viero, Spartà AM, Quattrone A, Provenzani A (2012) Downregulation of HuR as a new mechanism of doxorubicin resistance in breast cancer cells. Molecular cancer 11:

13. https://doi.org/10.1186/1476-4598-11-13 
Li H, Liu XQ, Chen XY (2005) Using gene chip technology to investigate the mechanism of aconitine's reversing the drug resistance of the KBV_(200) Cell. Chinese Journal of Information on Tcm

Li T-K, Liu LF (2001) Tumor cell death induced by topoisomerase-targeting drugs. Annual Review of Pharmacology and Toxicology 41: 53-77. https://doi.org/10.1146/annurev.pharmtox.41.1.53

Liang X, Chen L, Song L, Fei W, He M, He C, Yin Z (2017) Diterpenoid alkaloids from the root of Aconitum sinchiangense W. T. Wang with their antitumor and antibacterial activities. Nat Prod Res 31: 2016-2023. https://doi.org/10.1080/14786419.2016.1272113

Liang X, Chen N, Gao Y, He C, Yin Z, Zou Y, Yin L, Song X (2016a) Preparation and application of 8-lipoditerpenoid alkaloids. Chinese Patent: 201610776382.

Liang X, Gao Y, Luan S (2018) Two decades of advances in diterpenoid alkaloids with cytotoxicity activities. RSC Advances 8: 23937-23946. https://doi.org/10.1039/C8RA03911A

Liang X, Wu Q, Luan S, Yin Z, He C, Yin L, Zou Y, Yuan Z, Li L, Song X (2019) A comprehensive review of topoisomerase inhibitors as anticancer agents in the past decade. Eur J Med Chem 171: 129-168. https://doi.org/10.1016/j.ejmech.2019.03.034

Liang Y, Wu JL, Li X, Guo MQ, Leung LH, Zhou H, Liu L, Li N (2016b) Anti-cancer and Anti-inflammatory New Vakognavine-type Alkaloid from the Roots of Aconitum carmichaelii. Tetrahedron Letters 57: 58815884. https://doi.org/10.1016/j.tetlet.2016.11.065

Luan S, Gao Y, Liang X, Zhang L, Jing B (2021) Synthesis and structure-activity relationship of lipoditerpenoid alkaloids with potential target of topoisomerase lla for breast cancer treatment. Bioorganic Chemistry 109: 104699. https://doi.org/10.1016/j.bioorg.2021.104699

McClendon AK, Osheroff N (2007) DNA topoisomerase II, genotoxicity, and cancer. Mutation Research/Fundamental and Molecular Mechanisms of Mutagenesis 623: 83-

97. https://doi.org/10.1016/j.mrfmmm.2007.06.009

Mechetner E, Kyshtoobayeva A, Zonis S, Kim H, Stroup R, Garcia R, Parker RJ, Fruehauf JP (1998) Levels of multidrug resistance (MDR1) P-glycoprotein expression by human breast cancer correlate with in vitro resistance to taxol and doxorubicin. Clinical Cancer Research 4: 389-398.

Mingxin Tang, Sun G (1986) Aconitine's anti-tumor and anti-metastasis research and the observation of cancer treatment Beijing Journal of Traditional Chinese Medicine 27-28.

Mistry AR, Felix CA, Whitmarsh RJ, Annabel M, Andreas R, Bruno C, Anne P, Christoph W, Wiemels JL, Segal MR (2005) DNA topoisomerase II in therapy-related acute promyelocytic leukemia. New England Journal of Medicine 352: 1529-1538. https://doi.org/10.1056/NEJMoa042715 
Morris GM, Huey R, Lindstrom W, Sanner MF, Belew RK, Goodsell DS, Olson AJ (2009) AutoDock4 and AutoDockTools4: Automated docking with selective receptor flexibility. J Comput Chem 30: 2785-

2791. https://doi.org/10.1002/jcc.21256

Nitiss JL (2009a) DNA topoisomerase II and its growing repertoire of biological functions. Nature Reviews Cancer 9: 327-337. https://doi.org/10.1038/nrc2608

Nitiss JL (2009b) Targeting DNA topoisomerase II in cancer chemotherapy. Nature Reviews Cancer 9: 338-350. https://doi.org/10.1038/nrc2607

Pierce LC, Salomon-Ferrer R, Augusto F. de Oliveira C, McCammon JA, Walker RC (2012) Routine access to millisecond time scale events with accelerated molecular dynamics. Journal of Chemical Theory and Computation 8: 2997-3002. https://doi.org/10.1021/ct300284c

Pommier Y (2006) Topoisomerase I inhibitors: camptothecins and beyond. Nat Rev Cancer 6: $789-802$. https://doi.org/10.1038/nrc1977

Qiuping Xu , Jinghan Liu, Liu B (2016) Progress in Study on Antitumor Activity of C19-, C20-Diterpenoid Alkaloids. Progress in Pharmaceutical Sciences 40: 3-10.

Salomon-Ferrer R, Götz AW, Poole D, Le Grand S, Walker RC (2013) Routine microsecond molecular dynamics simulations with Amber on GPUs. 2. Explicit solvent particle mesh Ewald. Journal of Chemical Theory and Computation 9: 3878-3888. https://doi.org/10.1021/ct400314y

Sanner MF (1999) Python: a programming language for software integration and development. J Mol Graph Model 17: 57-61.

Sauer LA, Dauchy RT, Blask DE (2000) Mechanism for the antitumor and anticachectic effects of n-3 fatty acids. Cancer Research 60: 5289-5295.

Schmitta F, Golda M, Rothemunda M, Andronacheb IC, Biersacka B, Rainer Schoberta, Mueller T (2019) New naphthopyran analogues of LY290181 as potential tumor vascular-disrupting agents. European Journal of Medicinal Chemistry 163: 160-168. https://doi.org/10.1016/j.ejmech.2018.11.055

Schoeffler AJ, Berger JM (2008) DNA topoisomerases: harnessing and constraining energy to govern chromosome topology. Quarterly Reviews of Biophysics 41: 41-

101. https://doi.org/10.1017/S003358350800468X

Shatha AbuHammad, Zihlif M (2013) Gene expression alterations in doxorubicin resistant MCF7 breast cancer cell line. Genomics 101: 213-220. https://doi.org/10.1016/j.ygeno.2012.11.009

Sung H, Ferlay J, Siegel RL, Laversanne M, Soerjomataram I, Jemal A, Bray F (2021) Global Cancer Statistics 2020: GLOBOCAN Estimates of Incidence and Mortality Worldwide for 36 Cancers in 185 Countries. CA Cancer J Clin 71: 209-249. https://doi.org/10.3322/caac.21660

Page $15 / 22$ 
Trott O, Olson AJ (2010) AutoDock Vina: improving the speed and accuracy of docking with a new scoring function, efficient optimization, and multithreading. J Comput Chem 31: 455461. https://doi.org/10.1002/jcc.21334

Wada K (2012) Chapter 7 - Chemistry and Biological Activity of Diterpenoid Alkaloids. In: Atta ur R (ed.) Studies in Natural Products Chemistry. Elsevier, pp. 191-223. https://doi.org/10.1016/B978-0-444-59530$0.00007-1$

Wada K, Chiba R, Kanazawa R, Matsuoka K, Suzuki M, Ikuta M, Goto M, Yamashita H, Lee K-H (2015a) Six new norditerpenoid alkaloids from Delphinium elatum. Phytochemistry Letters 12: 79-

83. https://doi.org/10.1016/j.phytol.2015.02.010

Wada K, Hazawa M, Takahashi K, Mori T, Kawahara N, Kashiwakura I (2011) Structure-activity relationships and the cytotoxic effects of novel diterpenoid alkaloid derivatives against A549 human lung carcinoma cells. J Nat Med 65: 43-49. https://doi.org/10.1007/s11418-010-0452-3

Wada K, Ohkoshi E, Morrisnatschke SL, Bastow KF, Lee KH (2012) Cytotoxic esterified diterpenoid alkaloid derivatives with increased selectivity against a drug-resistant cancer cell line. Bioorganic \& Medicinal Chemistry Letters 22: 249-252. https://doi.org/10.1016/j.bmcl.2011.11.026

Wada K, Ohkoshi E, Zhao Y, Goto M, Morrisnatschke SL, Lee KH (2015b) Evaluation of Aconitum diterpenoid alkaloids as antiproliferative agents. Bioorg Med Chem Lett 25: 1525-1531.

Wada K. YH ( 2019) Cytotoxic Effects of Diterpenoid Alkaloids Against Human Cancer Cells. Molecules 24: 2317. https://doi.org/10.3390/molecules24122317

Wang J, Wang W, Kollman PA, Case DA (2006) Automatic atom type and bond type perception in molecular mechanical calculations. Journal of molecular graphics and modelling 25: 247260. https://doi.org/10.1016/j.jmgm.2005.12.005

Wang J, Wolf RM, Caldwell JW, Kollman PA, Case DA (2004) Development and testing of a general amber force field. J Comput Chem 25: 1157-1174.

Yamashita H, Miyao M, Hiramori K, Kobayashi D, Suzuki Y, Mizukami M, Goto M, Lee K-H, Wada K (2020) Cytotoxic diterpenoid alkaloid from Aconitum japonicum subsp. subcuneatum. Journal of Natural Medicines 74: 83-89. https://doi.org/10.1007/s11418-019-01346-z

Yamashita H, Takeda K, Haraguchi M, Abe Y, Kuwahara N, Suzuki S, Terui A, Masaka T, Munakata N, Uchida M, Nunokawa M, Kaneda K, Goto M, Lee K-H, Wada K (2018) Four new diterpenoid alkaloids from Aconitum japonicum subsp. subcuneatum. Journal of Natural Medicines 72: 230-

237. https://doi.org/10.1007/s11418-017-1139-9

Yamashita H. MM, Hiramori K., Kobayashi D., Suzuki Y., Mizukami M. , Goto M. ,Lee K.-H. , Wada K. (2020) Cytotoxic diterpenoid alkaloid from Aconitum japonicum subsp. subcuneatum. Journal of Natural 


\section{Tables}

Table 1 The $\mathrm{IC}_{50}(\mu \mathrm{M})$ results of compounds 1 and 4 against MCF-7, MCF-7/ADR and NIH3T3

\begin{tabular}{llll}
\hline No. & MCF-7 & MCF-7/ADR & NIH3T3 \\
\hline $\mathbf{1}$ & $7.58 \pm 0.06^{\mathrm{a}, \mathrm{b}}$ & $7.02 \pm 0.12^{\mathrm{a}, \mathrm{b}}$ & $72.08 \pm 0.15$ \\
$\mathbf{4}$ & $\mathbf{4 4 0 0}$ & $86.35 \pm 0.32^{\mathrm{a}, \mathrm{b}, \mathrm{c}}$ & - \\
\hline Adriamycin & $1.22 \pm 0.43^{\mathrm{a}, \mathrm{b}, \mathrm{c}}$ & $67.61 \pm 6.50^{\mathrm{b}, \mathrm{C}}$ & - \\
\hline Etoposide & $18.01 \pm 1.64^{\mathrm{a}, \mathrm{b}, \mathrm{c}}$ & $35.48 \pm 0.29^{\mathrm{a}, \mathrm{C}}$ & - \\
\hline SI & 9.51 & - & 10.26 \\
\hline
\end{tabular}

Note: Compared with positive control group ( ${ }^{\mathrm{a} A d r i a m y c i n, ~} \mathrm{~b}_{\text {Etoposide) }}$ ${ }^{\mathrm{a}} \mathrm{P}<0.05,{ }^{\mathrm{b}} \mathrm{P}<0.05$; Compared with 1 group, ${ }^{\mathrm{c}} \mathrm{P}<0.05$; - means no data, SI: selectivity of compound 1 between MCF-7 or MCF-7/ADR and NIH3T3 cells.

Table 2 Docking results of aconitine linoleate (1) and receptor protein molecules

\begin{tabular}{|c|c|c|c|}
\hline Receptor protein & PDB Number & Libdockscore & Poses \\
\hline Topo & $5 \mathrm{bTc}$ & 324.932 & 26 \\
\hline Topo $]$ & $1 \mathrm{nh} 3$ & 83.3349 & 1 \\
\hline Epidermal Growth Factor HER2 & 3WSQ & 147 & 23 \\
\hline Epidermal Growth Factor HER3 & 5040 & 142 & 93 \\
\hline Matrix metalloproteinases & $1 \mathrm{bqo}$ & 109.144 & 1 \\
\hline human basic fibroblast growth factor & $1 \mathrm{bfb}$ & 0 & 0 \\
\hline Human vascular endothelial growth factor & $1 \mathrm{vpf}$ & 0 & 0 \\
\hline Protein kinase $\mathrm{C}$ & $2 \mathrm{kf9}$ & 0 & 0 \\
\hline Insulin receptor & 3ekk & 0 & 0 \\
\hline Platelet-derived growth factor receptor & $3 \mathrm{mjg}$ & 144.6 & 56 \\
\hline Cytochrome P450 3A4 & $4 i 3 q$ & 133.45 & 4 \\
\hline Angiogenin 1 & $4 \mathrm{k} 0 \mathrm{v}$ & 138.278 & 16 \\
\hline Angiogenin 2 & $4 \mathrm{iml}$ & 132.827 & 32 \\
\hline Human tyrosine kinase c-KIT & $6 \mathrm{gqj}$ & 121.48 & 7 \\
\hline Human cyclooxygenase & $5 \mathrm{f} 19$ & 0 & 0 \\
\hline Epidermal growth factor receptor & 1ivo & 120 & 22 \\
\hline
\end{tabular}

Note: LibDockscore indicates the degree of docking between receptors and ligands; Poses represents the way ligands bind to proteins; If the score and poses are 0 , the docking failed.

Table 3 Comparation of the docking results of between aconitine linoleate (1) and aconitine (4) with receptor protein Topo $\square \alpha$ and Topo $\square \beta$

\begin{tabular}{lllll}
\hline Compound & Receptor protein & PDB Number & Libdockscore & Poses \\
\hline $\mathbf{1}$ & Topo $\square \alpha$ & $4 \mathrm{fm} 9$ & 102.535 & 13 \\
& Topo $\square \beta$ & $4 \mathrm{~g} 0 \mathrm{w}$ & 0 & 0 \\
\hline $\mathbf{4}$ & Topo $\square \alpha$ & $4 \mathrm{fm} 9$ & 0 & 0 \\
& Topo $\square \beta$ & $4 \mathrm{~g} 0 \mathrm{w}$ & 0 & 0 \\
\hline
\end{tabular}


Note: LibDockscore indicates the degree of docking between receptors and ligands; Poses represents the way ligands bind to proteins; If the score and poses are 0 , the docking failed.

Table 4. Average binding free energies $\left(\mathrm{kcal} \mathrm{mol}^{-1}\right)$ for the Topo2 $\alpha-1$ complex along with the different energy contributions

\begin{tabular}{llllllllll}
\hline Compd & $\Delta$ Evdw & $\Delta$ Eele & $\Delta$ Ggas & $\Delta$ EGB & $\Delta$ GSA & $\Delta$ Gsol & $\Delta \mathrm{H}$ & T $\Delta \mathrm{S}$ & $\Delta$ Gbind \\
\hline L29 & -99.8 & -45.6 & -145.4 & 88.7 & -11.0 & 77.7 & -67.7 & -58.6 & -9.1 \\
\hline
\end{tabular}

Table 5. Acute toxicity test results of compound 1 and 4 in mice through celiac injection

\begin{tabular}{llll}
\hline Compound & Concentration $(\mathrm{mg} / \mathrm{Kg})$ & Number of deaths & $\mathrm{LD}_{50}(\mathrm{nmol} / \mathrm{Kg})$ \\
\hline $\mathbf{1}$ & 180 & 3 & \\
& 190 & 4 & \\
\hline & 200 & 6 & \\
\hline & 210 & 7 & $2.2 \times 10^{5}$ \\
\hline $\mathbf{4}$ & 220 & 9 & \\
\hline & 0.3 & & \\
\hline & 0.4 & 1 & \\
\hline & 0.5 & 2 & $4.5 \times 10^{2}$ \\
\hline
\end{tabular}

Note: A total of 11 groups including the negative control group (dilute hydrochloric acid solution, pH 5.4), each with 10 mice; There was no death in the negative control group.

\section{Figures}




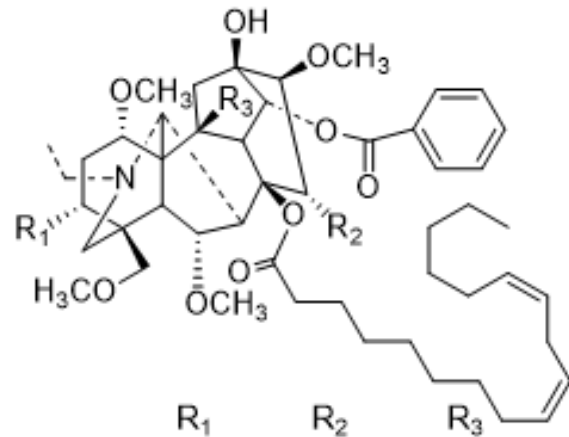

$1 \mathrm{OH} \quad \mathrm{OH}$

$2 \mathrm{H} \quad \mathrm{OH}$

$3 \mathrm{OH} \quad \mathrm{OH}$

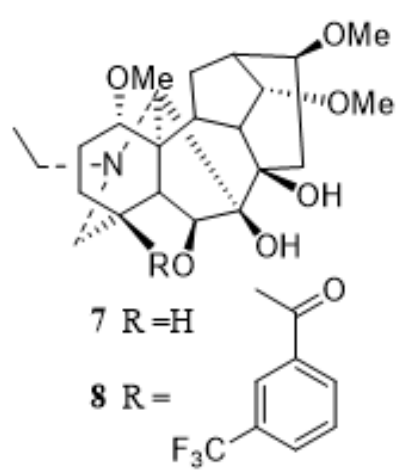

$\mathrm{H}$

$\mathrm{H}$

$\mathrm{OH}$

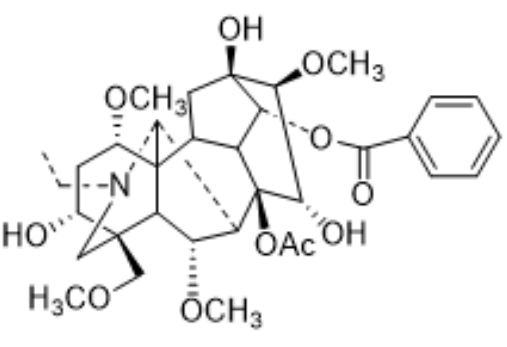

4
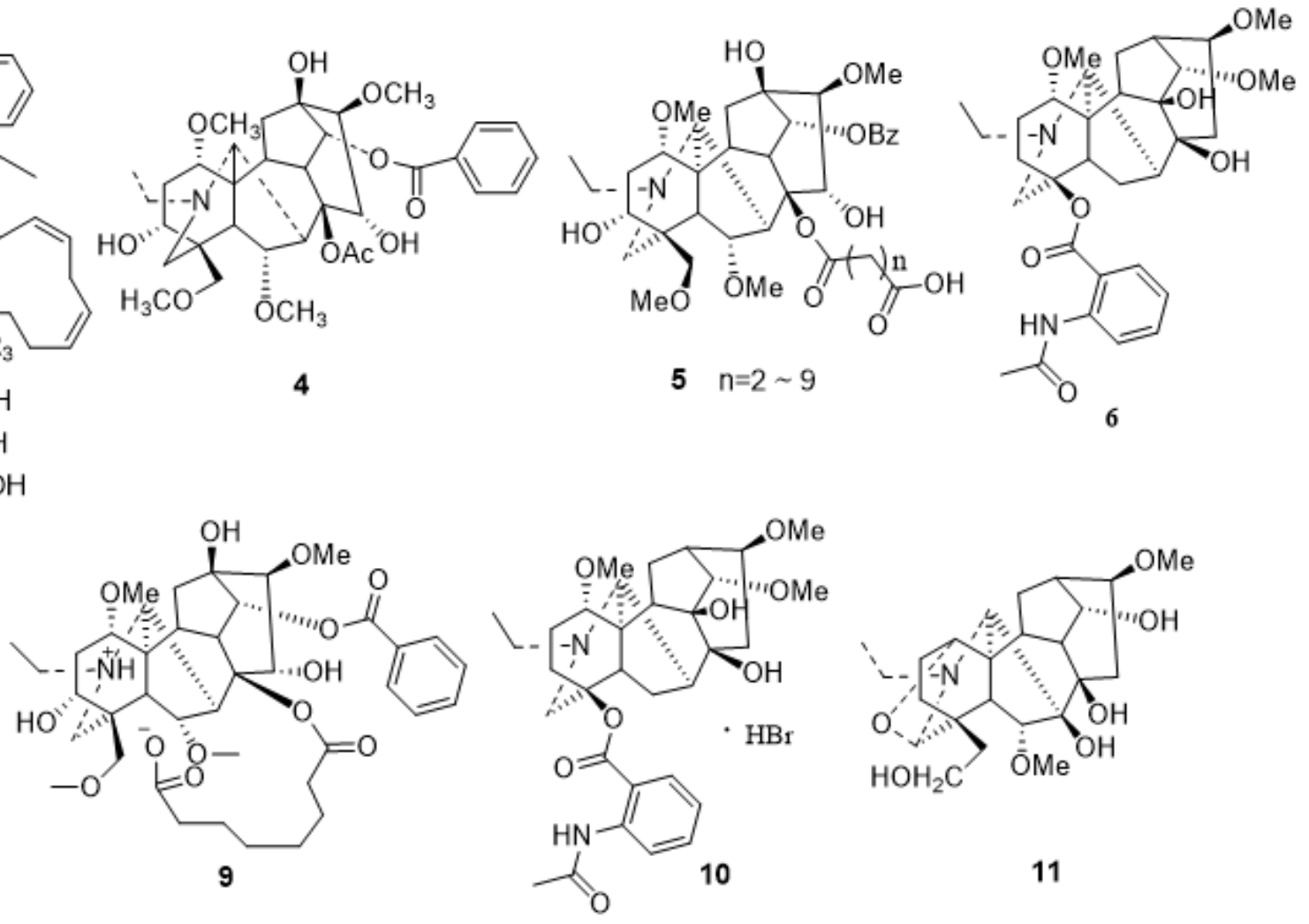

11

\section{Figure 1}

Part of the reported studies of C19-diterpenoid alkaloids with cytotoxicity
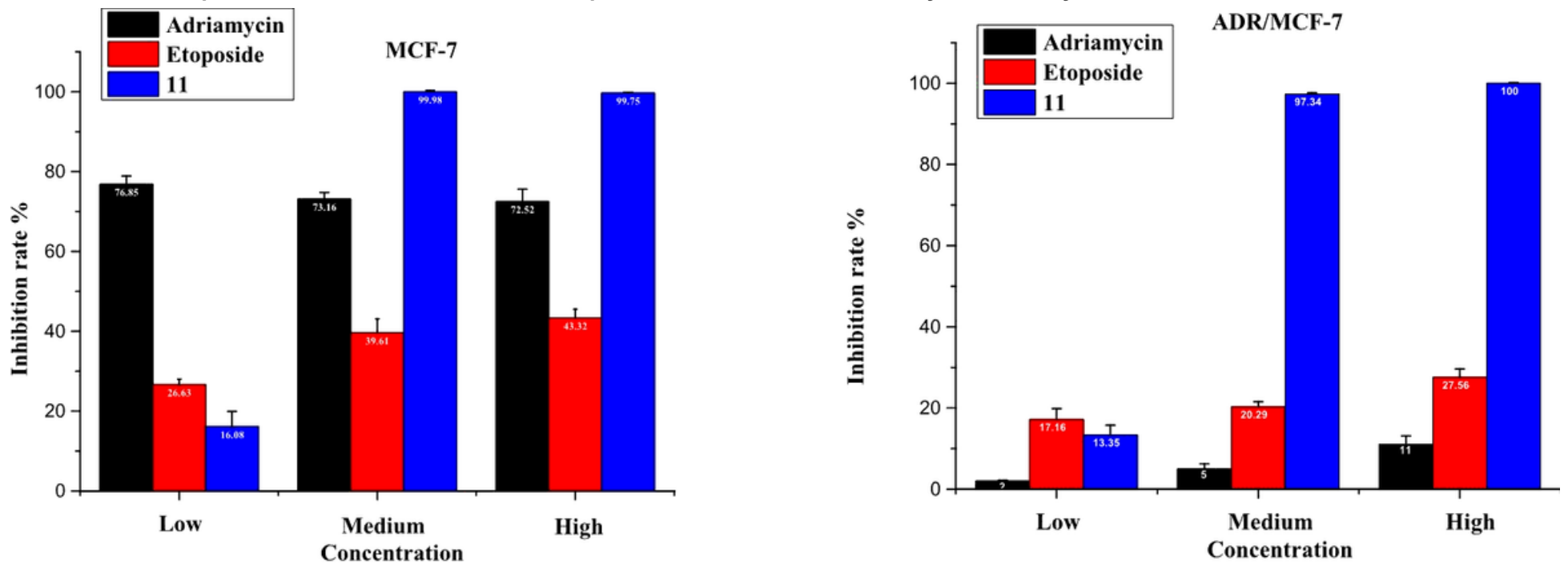

Figure 2

The in vitro anti-proliferative activity of Aconitine linoleate (1), adriamycin and etoposide examined against MCF-7 (Breast Cancer) and MCF-7/ADR cell lines. (Low: $5 \mu \mathrm{M}$, Medium: $10 \mu \mathrm{M}$, High: $15 \mu \mathrm{M}$ ) 


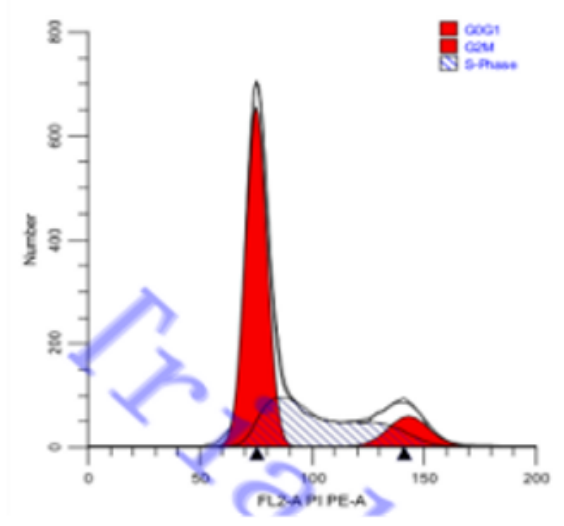

blank control group

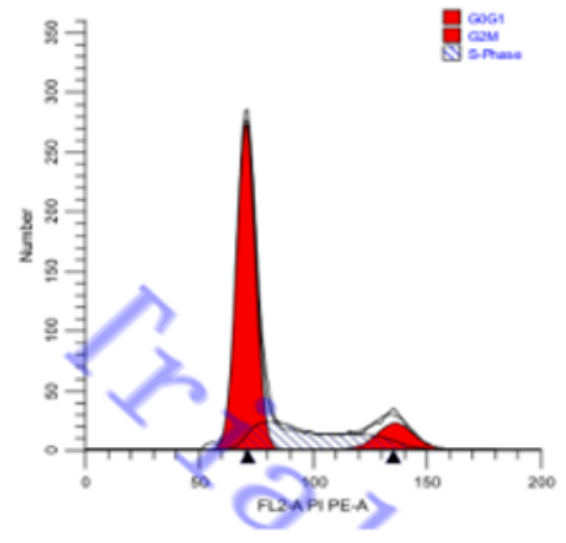

Syne Wizard Moded

File: 29-4 ites

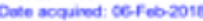
Dide andyred of et-2018 Auto Lireasity: Nob

cosis

Position. Flooting

Oas:

2067 Mean 13590 Ge261: 1.99

snace $R$.

$25.06 \%$ Mean on 15

Comanntere 1 11.74 \&

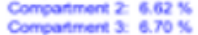

Modaled Everts: 4269

acs 1.111

Figure 3

Effect of aconitine linoleate (1) on the proliferation cycle of MCF-7/ADR cell

A
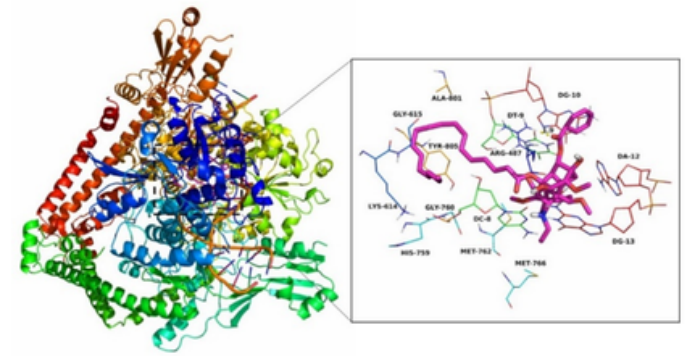

B

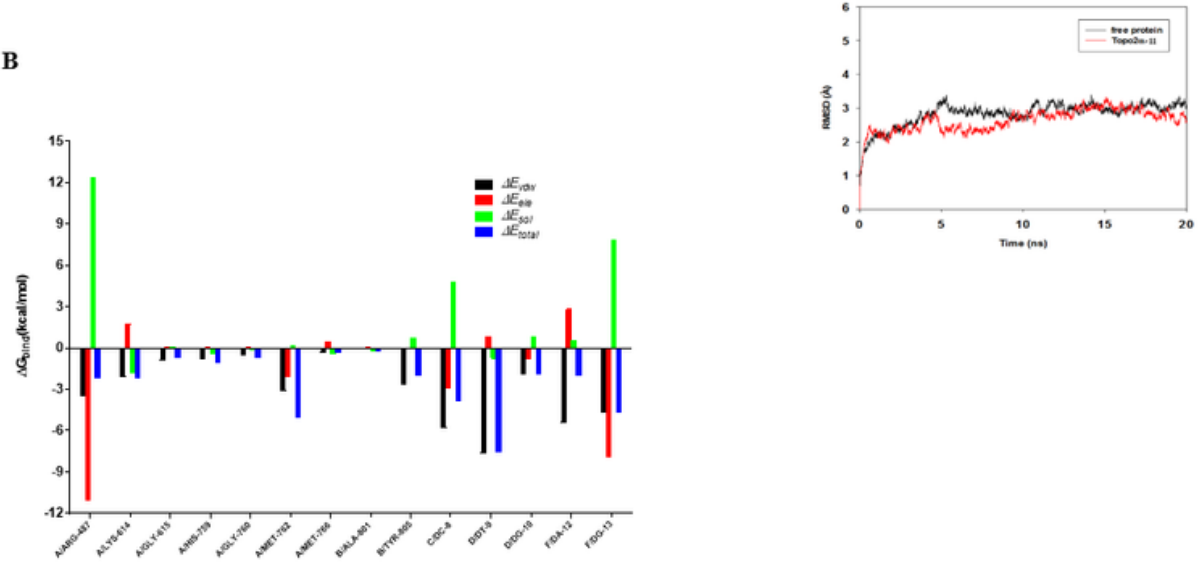

C aconitine linoleate group

D

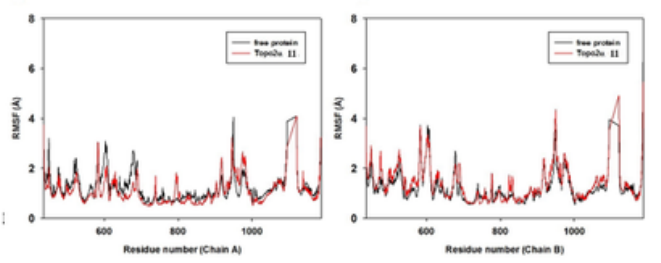

Figure 4 
Topo $₫$ a protein molecules. A) The predicted binding mode of 1 in Topo lla binding pocket obtained from MD simulation. B) Decomposition of the binding energy on a per-residue basis in the Topo lla-1 complex. C) The root-mean-square deviations (RMSDs) of all the atoms of Topo lla-1 complex with respect to its initial structure as function of time. D) RMSF of residues of the whole protein in Topo lla-1 complex and free Topo lla during the 20 ns simulation.

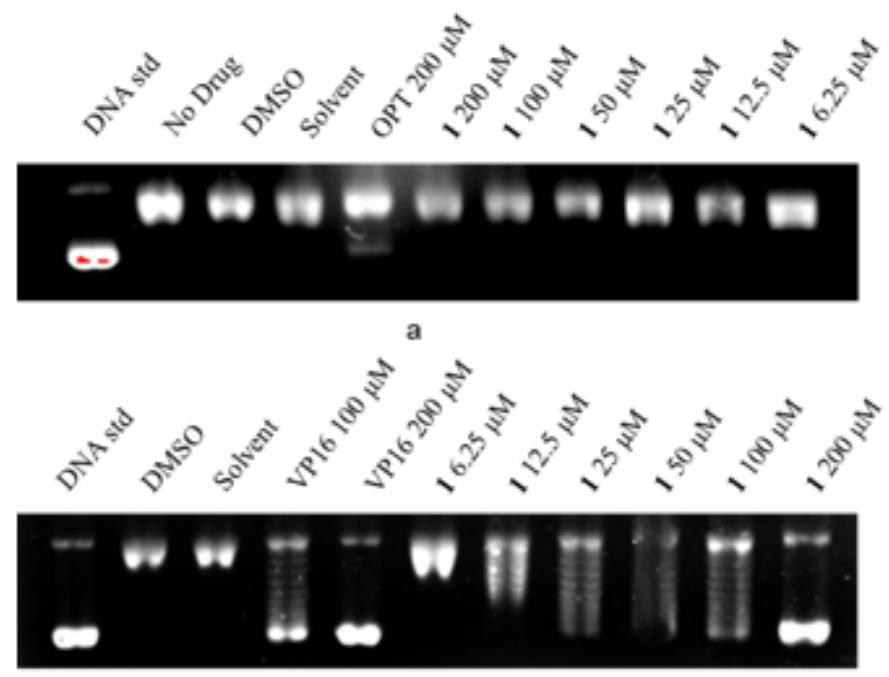

b

Figure 5

The Topoisomerase activity inhibition of aconitine linoleate (1). (a) Aconitine linoleate (1) inhibited the Topo $\triangle$ mediated pBR322 DNA relaxation; (b) Aconitine linoleate (1) inhibited the Topo $₫$ a mediated pBR322 DNA relaxation.

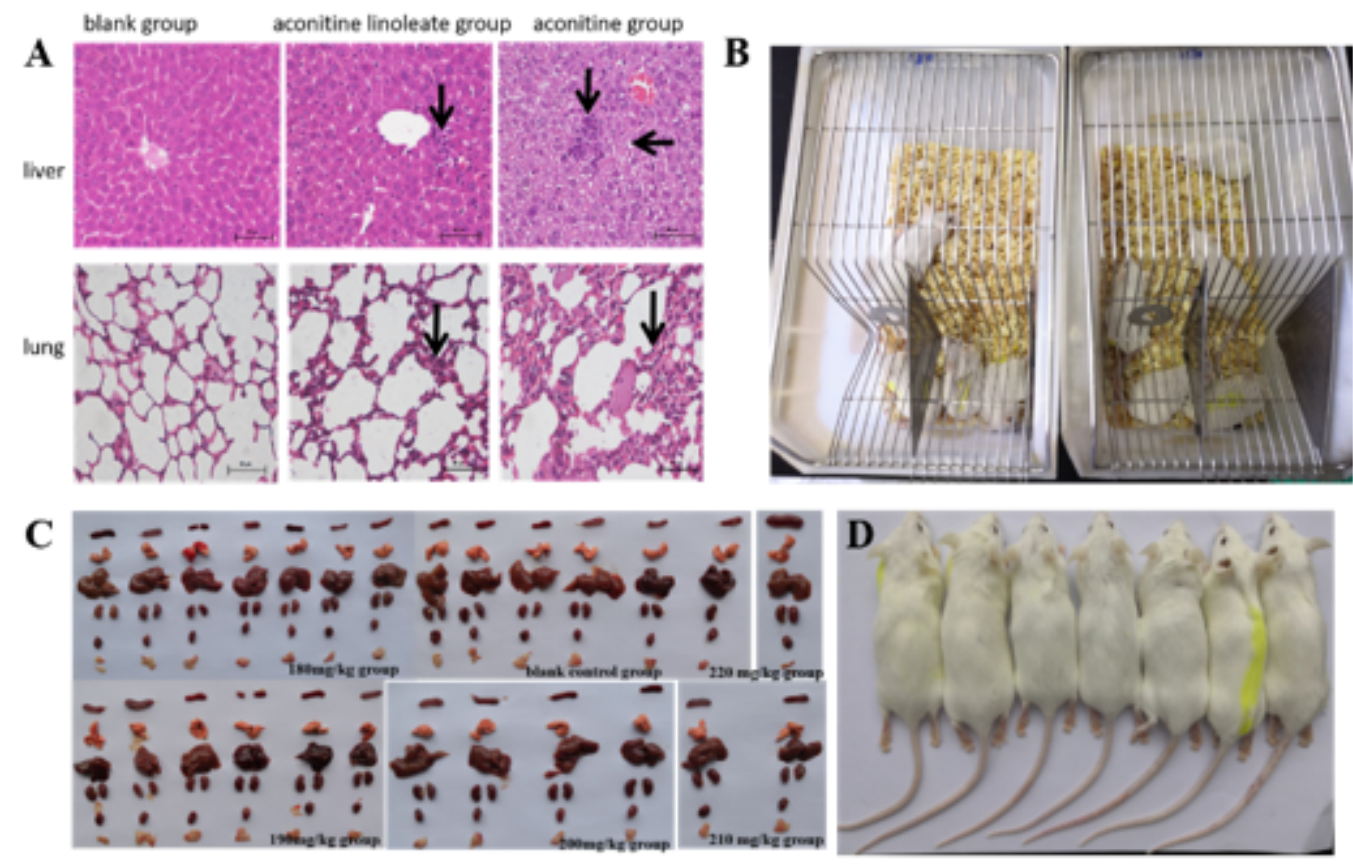




\section{Figure 6}

The acute toxicity of aconitine linoleate (1) on mice. A) Histological analysis of the liver and lung tissues from acute toxicity test model (blank control group; $180 \mathrm{mg} / \mathrm{kg}$ for aconitine linoleate group; $0.2 \mathrm{mg} / \mathrm{kg}$ for aconitine group). Photomicrograph of tissue stained with H\&E staining, $\times 400$. B-D). The vertical arrows on liver figures indicate vacuolar degeneration and the horizontal arrow indicates necrosis; the arrows on lung figures indicate the alveolar septum thickening. B) Acute toxicity test with $1(180 \mathrm{mg} / \mathrm{Kg}$ group) in mice through celiac injection, $n=10$. C) The main tissue of aconitine linoleate (1) in different concentration (180, 190, 200, 210, $220 \mathrm{mg} / \mathrm{Kg}$ ) and control group. D) The mice carcass after $14 \mathrm{~d}(180$ $\mathrm{mg} / \mathrm{Kg}$ group). (Note: The vertical arrows in A indicate vacuolar degeneration and the horizontal arrow indicates necrosis in liver and the alveolar septum thickening in lung, respectively.)

\section{Supplementary Files}

This is a list of supplementary files associated with this preprint. Click to download.

- MCF7Compound1.xls

- MCF7adriamycin.xls

- MCF7etoposide.xls

- MCF7ADRCompound1.xls

- MCF7ADRadriamycin.xls

- MCF7ADRetoposide.xls

- NIH3T3Compound1.xls

- SIJE.doc

- Onlinefloatimage1.png 